\title{
Flavor hierarchy at the chemical freeze-out from UrQMD
}

\author{
Tom Reichert ${ }^{1,4, *}$, Gabriele Inghirami ${ }^{2}$, and Marcus Bleicher ${ }^{1,2,3,4}$ \\ ${ }^{1}$ Institut für Theoretische Physik, Goethe Universität Frankfurt, Max-von-Laue-Strasse 1, D-60438 \\ Frankfurt am Main, Germany \\ ${ }^{2}$ GSI Helmholtzzentrum für Schwerionenforschung GmbH, Planckstr. 1, 64291 Darmstadt, Germany \\ ${ }^{3}$ John von Neumann-Institut für Computing, Forschungzentrum Jülich, 52425 Jülich, Germany \\ ${ }^{4}$ Helmholtz Forschungsakademie Hessen für FAIR (HFHF), GSI Helmholtzzentrum für Schwerionen- \\ forschung, Campus Frankfurt, Max-von-Laue-Str. 12, 60438 Frankfurt, Germany
}

\begin{abstract}
We introduce a novel approach based on elastic and inelastic scattering rates to extract the hyper-surface of the chemical freeze-out from a hadronic transport simulation. We use the Ultra-relativistic Quantum Molecular Dynamics (UrQMD) model to extract the chemical freeze-out hyper-surface of pions and kaons in the energy range from $E_{\text {lab }}=1.23 \mathrm{~A} \mathrm{GeV}$ to $\sqrt{s_{\mathrm{NN}}}=7.7 \mathrm{GeV}$. By employing a coarse-graining procedure, we can extract the local temperature $T$ and baryo-chemical potential $\mu_{\mathrm{B}}$ on the chemical freeze-out surface and compare them to results from statistical model analysis. We find good agreement between the pion chemical freeze-out line extracted from the simulation and the freeze-out line from the statistical model extracted from data. In addition the simulations also hint towards the existence of a flavor hierarchy similar to the one observed in recent lattice QCD calculations.
\end{abstract}

\section{Introduction}

There is an ongoing debate about whether the phase transition line is connected to the chemical freeze-out line, i.e. the stage at which flavor changing reactions cease and the chemical yields of the hadrons are fixed. On the one hand there is the thermal model utilizing a relativistic hadron gas described by a volume $(V)$, a temperature $(T)$ and the chemical potentials $\left(\mu_{i}\right)$ which successfully describes final state hadron yields over a broad range energies [1]. This finding is usually interpreted as thermal/chemical equilibrium being formed via statistical hadronization from the Quark-Gluon-Plasma (QGP). On the other hand there are ab initio lattice calculations investigating the temperature dependence of susceptibility ratios pointing towards a separation in the chemical decoupling temperatures of light and strange quarks [2-4].

In this article, we aim to contribute to the discussion of the "flavor hierarchy" question by extracting the microscopic chemical freeze-out hyper-surface $\Sigma_{\mu}^{\text {cfo }}\left(x^{\nu}\right)$ from a dynamical hadronic transport simulation without the need to fit measured particle yield. Extending our previous calculations for pions (as the dominant light hadron species) [5], this article addresses in addition the chemical freeze-out of strangeness via $K$ mesons. As originally pointed out by Pomeranchuk, the chemical or kinetic freeze-out of a system is determined

\footnotetext{
*e-mail: treichert@itp.uni-frankfurt.de
} 
by the competition between the scattering rate $\tau_{\text {scat }}$ and the expansion rate $\tau_{\exp }$ [6]. We thus aim to trace every final state hadron back to the space-time point of its first creation. Finally, we employ a coarse-graining procedure to infer the local temperature and baryo-chemical potential.

\section{Modeling chemical freeze-out in UrQMD}

The present study employs the Ultra-relativistic Quantum Molecular Dynamics (UrQMD v3.4) [7, 8] transport model in cascade mode. UrQMD is a hadron cascade model that simulates the dynamical evolution of heavy ion collisions by propagating the individual hadrons, modeling their interactions via the excitation of color flux-tubes (strings) and by further elastic and inelastic scatterings. A transition to a deconfined stage is not explicitly included in the cascade mode employed here.

Our procedure is based on the fact that during the hadronic phase (pseudo-elastic) reactions like $\Delta \leftrightarrow \pi N$ or $N^{*} \leftrightarrow K \Lambda$ effectively do not change the $\pi$ or $K$ yield over the lifetime of the intermediating resonance. Through investigation of the full time evolution of the UrQMD output, we can find every final state hadron and trace it back to the space-time point of its first creation. This defines the chemical freeze-out hyper-surface. For the details of the procedure we refer the reader to Ref. [5], where the chemical freeze-out hyper-surface of pions has been determined with UrQMD.

To relate thermodynamic quantities to the hyper-surface $\Sigma_{\mu}^{\text {cfo }}$, we coarse-grain the UrQMD simulations [9-11]. For the present calculation, a large ensemble of events is placed on a 4dimensional grid of resolution $\Delta t=0.25 \mathrm{fm} / \mathrm{c}$ and $\Delta x, y, z=1 \mathrm{fm}$. In each cell, the average energy-momentum tensor $T^{\mu v}$ and the average net-baryon current $j_{\mathrm{B}}^{\mu}$ is calculated. After a Lorentz-boost, their time-components represent the local energy density and the local netbaryon density in the Local Rest Frame (LRF). Finally, the energy and net-baryon density can be used to extract the local $T$ and $\mu_{\mathrm{B}}$ via interpolation of a Hadron Resonance Gas EoS [12].

\section{Results}

The results are obtained by analyzing central $\mathrm{Au}+\mathrm{Au}$ collisions from $E_{\mathrm{lab}}=1.23 \mathrm{~A} \mathrm{GeV}$ to $\sqrt{s_{\mathrm{NN}}}=7.7 \mathrm{GeV}$ calculated with the UrQMD model. The simulations are used as input to extract the chemical freeze-out coordinates and calculate the fields $\left(T(t, \vec{r}), \mu_{\mathrm{B}}(t, \vec{r})\right)$ with the aforementioned coarse-graining procedure. Central events are selected via an impact parameter cut of $b_{\max }=3.4 \mathrm{fm}$ at all investigated energies. We focus on the chemical freezeout of $\pi$ and $K$ which finally decouple in the volume with $\left|z_{\text {kin }}\right| \leq 5 \mathrm{fm}$.

\subsection{Freeze-out of pions versus kaons}

Let us start with the distributions of the chemical freeze-out times (left), temperatures (middle) and baryo-chemical potential (right) shown in Fig. 1 for kaons (solid lines) and pions (dotted lines) in central $\mathrm{Au}+\mathrm{Au}$ collisions from $E_{\mathrm{lab}}=1.23 \mathrm{~A} \mathrm{GeV}$ to $\sqrt{s_{\mathrm{NN}}}=7.7 \mathrm{GeV}$ calculated with the UrQMD model. In general, the freeze-out time, temperature and $\mu_{\mathrm{B}}$ distributions of pions and of kaons are all peaked indicating that chemical decoupling indeed happens at a rather specific stage of the system evolution, hence the extraction of the chemical freeze-out hyper-surface is reasonable. At very low energies the chemical freeze-out times of the pions and kaons are both peaked but separated by $2 \mathrm{fm} / \mathrm{c}$, however with increasing energy the shift between the distributions vanishes. This observation is expected a) due to the 

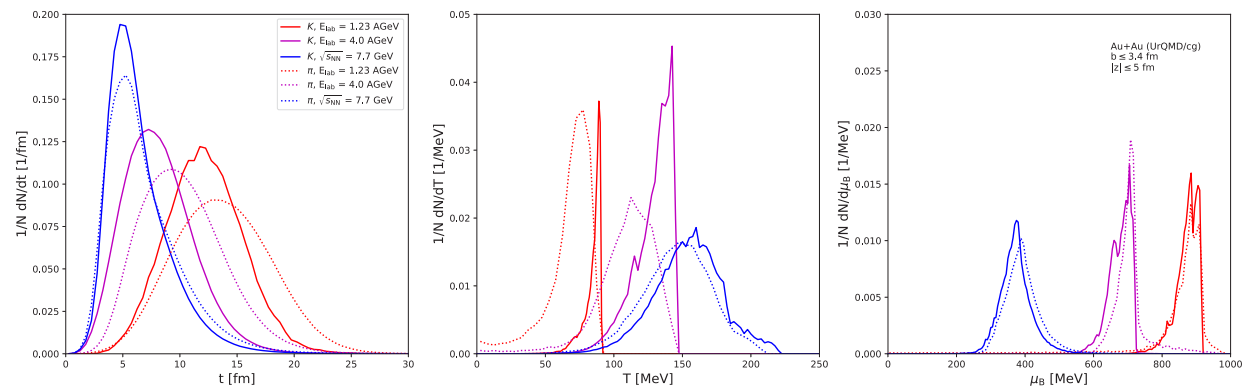

Figure 1. [Color online] Distributions of the chemical freeze-out times (left), of the chemical freeze-out temperatures (middle) and the chemical freeze-out baryo-chemical potentials (right) for kaons (solid) and pions (dotted) in central $\mathrm{Au}+\mathrm{Au}$ collisions from $E_{\text {lab }}=1.23 \mathrm{~A} \mathrm{GeV}$ to $\sqrt{s_{\mathrm{NN}}}=7.7 \mathrm{GeV}$ calculated with the UrQMD model.

fact that at low energies (low temperatures) strangeness production is canonically suppressed (also in transport simulations [13]) and furthermore b) due to the small $K$ absorption cross section on nuclear matter. This (low energy) separation in the average decoupling time is reflected in the extracted temperatures: pions decouple during a broader time span, thus at temperatures ranging from $40 \mathrm{MeV}$ to $100 \mathrm{MeV}$ while kaons, to the contrary, decouple in a narrow temperature slice around $80-90 \mathrm{MeV}$. This separation again vanishes with increasing energy. The average $\langle T\rangle$ moreover approaches $150 \mathrm{MeV}$ independently of the hadron species at the largest collision energy lining up with statistical model fits to measured hadron yields. The average baryo-chemical potential of both the pions and the kaons decreases with increasing energy due to the production of anti-baryons at higher energies. Here, the separation between $\pi$ and $K$ becomes small.

\subsection{Thermodynamics on the hyper-surface}

We are now in the position to visualize our results in the QCD phase diagram. A meaningful $T, \mu_{\mathrm{B}}$ tuple is obtained by averaging the distributions shown in the previous section. Fig. 2 shows the $T, \mu_{\mathrm{B}}$ combinations extracted from the chemical freeze-out of pions (black triangles up) and of kaons (red circles) and compares them to well established statistical models fits to experimental hadron yields (blue crosses) [1, 14-18]. In addition we also show results from Ref. [19] in which different ensembles of experimental hadron yields are fitted with the GCE as orange symbols and results from [20] (green symbols) in which the authors used strangeness susceptibilities to calculate flavor hierarchy at chemical freeze-out.

We observe that our results using pions are in very good agreement with the known statistical model fits to the available experimental data [14-18,21]. Our results on the chemical freeze-out hyper-surface of kaons show generally a larger temperature speaking for an earlier freeze-out. As already discussed, in our simulation (driven by scattering rates) the separation in chemical freeze-out temperature between light and strange flavors $\Delta T \equiv T^{K}-T^{\pi}$ decreases with increasing energy from $20 \mathrm{MeV}$ at HADES to $10 \mathrm{MeV}$ at medium RHIC energies, different from the results in $[4,19,20]$.

\section{Summary}

We employed a novel method to extract the microscopic chemical freeze-out hyper-surface from a dynamical hadronic transport simulation to investigate the flavor hierarchy. For this, 


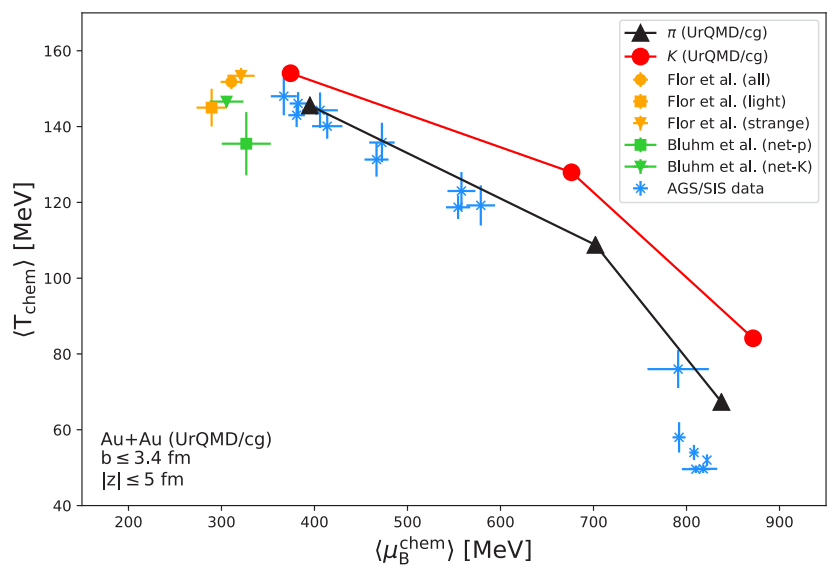

Figure 2. [Color online] The chemical freeze-out line $\left(T, \mu_{\mathrm{B}}\right)$ of pions (black) and kaons (red) in central $\mathrm{Au}+\mathrm{Au}$ collisions from $E_{\mathrm{lab}}=1.23 \mathrm{~A} \mathrm{GeV}$ to $\sqrt{s_{\mathrm{NN}}}=7.7 \mathrm{GeV}$ calculated with the UrQMD model. Also shown are fits to experimental hadron yields from AGS [14, 21] and SIS [15-18] as well as chemical freeze-out estimates using kaon fluctuations from [20] (green symbols) and using different hadron ensembles in thermal fits from [19] (orange symbols).

we used the Ultra-relativistic Quantum Molecular Dynamics (UrQMD) model in version 3.4 to calculate the chemical freeze-out hyper-surface of pions and of kaons from central $\mathrm{Au}+\mathrm{Au}$ collisions in the energy range from $E_{\mathrm{lab}}=1.23 \mathrm{~A} \mathrm{GeV}$ to $\sqrt{s_{\mathrm{NN}}}=7.7 \mathrm{GeV}$. The local temperature and baryo-chemical potential on this hyper-surface were extracted by coarse-graining the UrQMD output. Our calculations reveal that the statistical model data as well as flavor hierarchy in the chemical decoupling of pions and kaons can be well described by hadronic transport calculations. In contrast to previous results in the literature which investigated this phenomenon at very high energies (LHC, top RHIC), we observed that flavor separation increases with increasing energy at low collision energies (GSI, low RHIC).

The authors thank Jamie Karthein and Marcus Bluhm for fruitful discussion in the aftermath of the "Strangeness in Quark Matter 2021" conference. This work was supported in the framework of COST Action CA15213 THOR and we acknowledge support from STRONG-2020, financed by the Horizon 2020 program of the European Community. Computational resources were provided by the Center for Scientific Computing (CSC) of the Goethe University. G. Inghirami acknowledges funding by the Deutsche Forschungsgemeinschaft (DFG, German Research Foundation) - Project number 315477589 - TRR 211.

\section{References}

[1] A. Andronic, P. Braun-Munzinger, K. Redlich, J. Stachel, Nature 561, 321 (2018), 1710.09425

[2] R. Bellwied, S. Borsanyi, Z. Fodor, S.D. Katz, C. Ratti, Phys. Rev. Lett. 111, 202302 (2013), 1305.6297

[3] P. Alba, W. Alberico, R. Bellwied, M. Bluhm, V. Mantovani Sarti, M. Nahrgang, C. Ratti, Phys. Lett. B 738, 305 (2014), 1403.4903 
[4] J.M. Stafford, P. Alba, R. Bellwied, V. Mantovani-Sarti, J. Noronha-Hostler, P. Parotto, I. Portillo-Vazquez, C. Ratti, Springer Proc. Phys. 250, 367 (2020), 1912 . 12968

[5] T. Reichert, G. Inghirami, M. Bleicher, Eur. Phys. J. A 56, 267 (2020), 2007.06440

[6] G. Inghirami, T. Reichert, M. Bleicher (2021), 2106.04543

[7] S.A. Bass et al., Prog. Part. Nucl. Phys. 41, 255 (1998), nucl-th/9803035

[8] M. Bleicher et al., J. Phys. G 25, 1859 (1999), hep-ph/9909407

[9] G. Inghirami, P. Hillmann, B. Tomášik, M. Bleicher, J. Phys. G 47, 025104 (2020), 1909.00643

[10] P. Huovinen, M. Belkacem, P.J. Ellis, J.I. Kapusta, Phys. Rev. C 66, 014903 (2002), nucl-th/0203023

[11] S. Endres, H. van Hees, J. Weil, M. Bleicher, Phys. Rev. C 91, 054911 (2015), 1412.1965

[12] D. Zschiesche, S. Schramm, J. Schaffner-Bielich, H. Stoecker, W. Greiner, Phys. Lett. B 547, 7 (2002), nucl-th/0209022

[13] O. Fochler, S. Vogel, M. Bleicher, C. Greiner, P. Koch-Steinheimer, Z. Xu, Phys. Rev. C 74, 034902 (2006), nucl-th/0601062

[14] F. Becattini, M. Gazdzicki, A. Keranen, J. Manninen, R. Stock, Phys. Rev. C 69, 024905 (2004), hep-ph/0310049

[15] J. Cleymans, H. Oeschler, K. Redlich, Phys. Rev. C 59, 1663 (1999), nucl-th/9809027

[16] F. Becattini, J. Cleymans, A. Keranen, E. Suhonen, K. Redlich, Phys. Rev. C 64, 024901 (2001), hep-ph/0002267

[17] R. Averbeck, R. Holzmann, V. Metag, R.S. Simon, Phys. Rev. C 67, 024903 (2003), nucl-ex/0012007

[18] G. Agakishiev et al. (HADES), Eur. Phys. J. A 47, 21 (2011), 1010. 1675

[19] F.A. Flor, G. Olinger, R. Bellwied, Phys. Lett. B 814, 136098 (2021), 2009. 14781

[20] M. Bluhm, M. Nahrgang, Eur. Phys. J. C 79, 155 (2019), 1806.04499

[21] L. Bravina, A. Faessler, C. Fuchs, Z.D. Lu, E.E. Zabrodin, Phys. Rev. C 66, 014906 (2002), nucl-th/0206014 